19 Revue d'histoire du XIXe siècle

Société d'histoire de la révolution de 1848 et des

révolutions du XIXe siècle

$61 \mid 2020$

Dans l'intimité de l'exil

\title{
Bruno CHENIQUE, Citoyens du Monde. Noirs et Orientaux de Géricault
}

Alain Messaoudi

\section{(2) OpenEdition \\ Journals}

Édition électronique

URL : https://journals.openedition.org/rh19/7266

DOI : $10.4000 /$ rh 19.7266

ISSN : $1777-5329$

Éditeur

La Société de 1848

Édition imprimée

Date de publication : 1 décembre 2020

Pagination : 279-282

ISSN : 1265-1354

Référence électronique

Alain Messaoudi, «Bruno CHENIQUE, Citoyens du Monde. Noirs et Orientaux de Géricault », Revue

d'histoire du XIXe siècle [En ligne], 61 | 2020, mis en ligne le 20 janvier 2021, consulté le 20 août 2021.

URL : http://journals.openedition.org/rh19/7266 ; DOI : https://doi.org/10.4000/rh19.7266

Ce document a été généré automatiquement le 20 août 2021.

Tous droits réservés 


\title{
Bruno CHENIQUE, Citoyens du Monde. Noirs et Orientaux de Géricault
}

\author{
Alain Messaoudi
}

\section{RÉFÉRENCE}

Bruno CHENIQUE, Citoyens du Monde. Noirs et Orientaux de Géricault, Paris/Chalon-surSaône, Lienart Éditions/Musée Denon, 2020, 256 p., $35 €$.

1 Publié à l'occasion d'une exposition-dossier organisée autour d'un Portrait de Noir qui appartient aux collections du musée Denon à Chalon-sur-Saône, ce livre, fort bien illustré (145 figures) et solidement documenté (avec une riche bibliographie chronologiquement ordonnée), démontre de façon convaincante la centralité des figures du Noir et de l'Oriental dans l'œuvre de Géricault. Bruno Chenique explore depuis trois décennies l'univers de l'artiste, en soulignant le caractère révolutionnaire de son romantisme. Il poursuit donc ici un travail jalonné par plusieurs publications liées à des expositions, parmi lesquelles on peut citer la rétrospective présentée au musée des Beaux-Arts de Lyon en 2006, et celle centrée sur les études pour Le Radeau de la Méduse au musée d'Art Roger-Quillot de Clermont-Ferrand en 2012.

2 L'auteur se démarque d'une approche formaliste qui continue, selon lui, à dominer trop souvent le monde de l'histoire de l'art, avec pour effet de gommer la dimension politique des œuvres, et de les vider d'une grande partie de leur sens. Il rappelle ainsi, dans son avant-propos («Noirs et Orientaux: Modèles mais pas seulement»), que l'exposition Le Modèle noir de Géricault à Matisse, qui revendiquait une "approche multidisciplinaire, entre histoire de l'art et histoire des idées ${ }^{1} "$, n'était pas exempte de ce travers. Si cette exposition a marqué un tournant, de par le public nouveau qu'elle a attiré, elle a éludé les engagements de Géricault, dont Bruno Chenique, à travers les sept chapitres de son livre, rappelle la radicalité.

3 La première difficulté rencontrée par l'historien qui travaille sur Géricault tient à la définition du corpus de ses œuvres. Le premier chapitre du livre, « Le Portrait de Noir du 
musée Denon et les aventures du connoisseurship ", rappelle les enjeux des attributions (et désattributions), et les éléments souvent fragiles sur lesquels elles se fondent. Bruno Chenique en analyse la chronique pour le Portrait de Noir du musée Denon, en lequel il voit une étude réalisée vers 1811-1812, alors que Géricault était élève de l'atelier de Pierre Guérin. La toile aurait très vraisemblablement été achetée à la vente de l'atelier du peintre, en 1824, par Jean-Baptiste-Prudent Carbillet, élève de Gros, pour le cabinet de modèles de son oncle, Jacques-François Carbillet, directeur de l'école gratuite de dessin fondée trois ans plus tôt à Chalon-sur-Saône. Si l'inventaire après décès de Carbillet ne donne aucune indication sur l'auteur du tableau, ce dernier est présenté comme étant de la main de Géricault lorsqu'il intègre les collections du nouveau musée municipal en 1866. On suivra Bruno Chenique lorsqu'il suppose que cette affirmation reprenait un savoir transmis oralement, sans qu'il ait été alors besoin de preuves écrites.

Les cinq chapitres suivants forment le cœur de l'ouvrage. Ils rappellent l'importance de la figure du Noir dans l'œuvre de Géricault: «Le grand mélancolique qu'il était, cherchait peut-être dans l'Autre sa propre humanité afin de redéfinir son moi et l'ancrer dans une fraternité (lui, le fils unique) universelle» (p. 48). Ils replacent la quête personnelle de l'artiste dans des débats sur l'abolition de la traite et de l'esclavage (chapitre $2:$ "Racisme et colonialisme : Consulat, Empire et Restauration ») et soulignent que la figure du Noir est présente dans l'œuvre de Géricault bien avant l'élaboration du Radeau de la Méduse en 1818-1819 - on rappellera que c'est une figure de métis qui, debout, brandit l'étoffe blanche et rouge qui signale le salut (chap. 3 : " Géricault et la figure de l'altérité : une très lente redécouverte »). Plusieurs historiens de l'art, Bruno Chenique le rappelle, se sont certes intéressés à la présence politique des Noirs dans l'œuvre de Géricault (Frédéric-Henri Lem, qui identifia en 1962 des dessins du Carnet Zoubaloff datés de 1813-1814; Klaus Berger et Diane Chalmers Johnson, qui analysèrent en 1969 la lithographie des Boxeurs, inspirée de gravures populaires qui mettent en scène le combat d'un Blanc et d'un Noir). Mais d'autres, comme Lorenz Eitner ${ }^{2}$, ont refusé de la voir, au motif que l'artiste se serait placé au-dessus de la politique. Bruno Chenique, qui se démarque d'Eitner, identifie le couple de Noirs assis sur un rocher, représenté sur une aquarelle datée de 1817 et conservée dans un album inédit réapparu en 2008, comme les héros d'un roman abolitionniste de Jean-Baptiste Piquenard, Adonis, ou le bon nègre, anecdote coloniale (1798). Il rappelle que Géricault, grand lecteur, avait plusieurs amis liés à Haïti : Louis Bro, qui combattit dans l'armée de Leclerc ; Henri-Joseph de Forestier, natif de Saint-Domingue ; Auguste Lethière dont le père, le peintre Guillaume Guillon-Lethière, natif de Guadeloupe, était le fils naturel d'une esclave affranchie. Nommé en 1807 à la tête de la Villa Médicis, il y resta jusqu'en décembre 1816, et Géricault l'y rencontra. Bruno Chenique rappelle que GuillonLethière a offert en 1822 à la République d'Haïti, pour célébrer la réunification de l'île, son Serment des ancêtres, qui représente l'union en 1802 du mulâtre Alexandre Pétion et de l'ancien esclave affranchi Jean-Jacques Dessalines, afin de faire face au corps expéditionnaire envoyé par la France pour reconquérir l'île. Ce rapprochement leur a permis de défaire l'armée commandée par le général Leclerc, époux de Pauline Bonaparte, et de proclamer en 1804 l'indépendance d'Haïti (chap. 4: «Le retour du refoulé : "Splendeur et misère de la race noire" »). Le chapitre suivant ("Géricault et le mouvement abolitionniste ») montre comment la figure du Noir à genoux, les mains jointes en prière, poignets et cheville attachées par une chaîne, avec pour légende « $\mathrm{Ne}$ suis-je pas un homme, un frère?", largement diffusée à partir de 1787 par le 
mouvement abolitionniste, est réinterprétée par Géricault dans Le Radeau de la Méduse, sous la forme d'un symbole de fraternité, superposant mains noires et mains blanches. Bruno Chenique repère ensuite dans le tableau un écho subtil de la question de SaintDomingue, dont l'indépendance sera reconnue par la France en 1825 : croix de la Légion d'honneur, spectre d'un drapeau tricolore (l'auteur rappelle qu'en 1793 et 1794, des députés de couleur de Saint-Domingue envoyèrent au Club des Jacobins des drapeaux tricolores sur lequel étaient peints un Blanc, un mulâtre et un Noir), présence d'un Noir et d'un Blanc aux côtés des portraits des témoins de la tragédie, Corréard et Savigny (chap. 6: «Le Radeau de la méduse: une allégorie de Saint-Domingue»). Ce serait d'ailleurs après avoir achevé Le Radeau de la Méduse, en 1820, que Géricault aurait formé le projet d'une œuvre monumentale sur la Traite des Noirs, connue par un dessin (p. 117, p. 213 sq. et fig. 102).

Dans le dernier chapitre du livre ( Il parlait de visiter l'Orient »), la figure de l'Oriental est abordée plus rapidement. On y constate la fluidité (ou le flou) des qualificatifs (turc, arabe, algérien) en usage au XIX siècle lorsqu'il s'agit de qualifier Mustafa, qui servit de modèle à Horace Vernet, Girodet et Géricault, ce dernier l'ayant aussi employé comme domestique. En comparant son portrait réalisé par Girodet avec la copie qu'en fit Géricault, Bruno Chenique souligne combien cette dernière accentue l'étrangeté du regard, autre, voire aliéné. La figure du cheval arabe est illustrée, entre autres, par le dessin poignant d'un animal mourant, la tête soutenue par un homme coiffé d'un turban, destiné à la page de titre d'un manuel d'anatomie (1820-1821), et par une aquarelle figurant le Giaour de Byron (1820-1823). Autant d'éléments d'un dossier dont on peut espérer qu'il sera approfondi dans un prochain ouvrage.

6 Ce livre apporte une contribution importante à la connaissance des Noirs et des Orientaux en France dans les premières décennies du XIX ${ }^{e}$ siècle, des images qu'ils suscitent, et des débats sur leur place dans la sociétés. Il a pour qualité d'être fondé sur une documentation précise, l'auteur exposant sans détour le cheminement de son processus d'enquête, les controverses autour de l'attribution et de l'interprétation des œuvres, et les résistances rencontrées par des lectures qui les réinscrivent dans les débats politiques du temps. Il fait donc réellement dialoguer les apports des historiens de l'art et des historiens. Il rappelle aux premiers l'importance d'enquêtes permettant non seulement de documenter les œuvres mais aussi de reconstituer l'imaginaire social, la culture politique et les conceptions intellectuelles des artistes. Mais il devrait aussi montrer aux seconds l'importance des sources iconographiques, qui, bien utilisées, permettent de donner un éclairage nouveau à des sources écrites parfois lacunaires.

\section{NOTES}

1. Présentation générale de l'exposition sur le site du musée d'Orsay [https://www.museeorsay.fr/fr/evenements/expositions/aux-musees/presentation-generale/article/le-modelenoir-47692.html?tx_ttnews\%5BbackPid\%5D=649\&cHash=07204f3747] (consulté le 30 octobre 2020). 
2. Spécialiste de la peinture française du XIX siècle, et auteur d'une monographie de référence de Géricault, Lorenz Eitner (1919-2009) dirigea entre 1963 et 1989 le département des beaux-arts de l'Université de Stanford.

3. Il participe ainsi à un renouveau historiographique dont témoignent, par exemple deux ouvrages. Pour les Orientaux musulmans : Jocelyne Dakhlia, Wolfgang Kaiser (dir.), Les Musulmans dans l'histoire de l'Europe, t. 1 : Une intégration invisible; t. 2 : Passages et contacts en Méditerranée, Paris, Albin Michel, 2011, 2013 ; pour les Noirs : Julie Duprat, «Présences noires à Bordeaux : passage et intégration des gens de couleur à la fin du XVIII ${ }^{\mathrm{e}}$ siècle ", Thèse, École nationale des chartes, sous la direction d'olivier Poncelet, 2017. 